Минимально инвазивные технологии при заболеваниях печени,

желчных протоков и поджелуцочной железы

Minimally invasive techniques for liver, bile ducts and pancreas diseases

ISSN 1995-5464 (Print); ISSN 2408-9524 (Online)

https://doi.org/10.16931/1995-5464.2021-3-34-45

Портосистемные шунтируюшие операции

при осложненной портальной гипертензии:

современные возможности мини-инвазивных технологий

Хоронько Ю.В. *, Косовцев Е.В., Козыревский М.А., Хоронько Е.Ю., Криворотов Н.А., Чесноков В.В.

ФГБОУ ВО “Ростовский государственный медицинский университет” Министерства здравоохранения РФ; 344022, Ростов-на-Дону, пер. Нахичеванский, д. 29, Российская Федерация

Цель. Улучшить результаты лечения больных осложненной портальной гипертензией цирротического генеза применением операции TIPS, дополненной эндоваскулярной облитерацией путей притока к пищеводножелудочным варикозно расширенным венам.

Материал и методы. TIPS выполнено 172 пациентам с варикозным пищеводно-желудочным кровотечением. Больных распределили в три клинические группы. Шунтирующее пособие применили 62 больным. Еще 110 пациентам выполнили TIPS и селективную облитерацию путей притока к пищеводно-желудочным венам. Прослежены ближайшие и отдаленные результаты (до $140 \mathrm{Mec}$ ), частота тромбоза, рецидива кровотечения и летальность, а также их связь с установленными факторами риска осложнений.

Результаты. У всех пациентов достигнуто эффективное уменьшение портосистемного градиента давления и редукция проявлений портальной гипертензии. Рецидив кровотечения, вызванный тромбозом шунта, произошел у $23(13,3 \%)$ пациентов. В I клинической группе $(n=62)$ это осложнение отмечено у $9(14,5 \%)$ больных, во II $(n=54)-$ у $11(20,4 \%)$, в III $(n=56)$ - лишь у $3(5,4 \%)$. Выживаемость без кровотечения уменьшалась с 1,0 до 0,82 за 83,9 мес, затем переходила в плато. Резкое уменьшение кривой Каплана-Майера с 1,0 до 0,88 наблюдали в течение 24,5 мес после вмешательства. Наибольшее число летальных исходов было в I группе $(30,6 \%)$, наименьшее - в III группе $(7,1 \%)$.

Заключение. TIPS, дополненное облитерацией притоков к пищеводно-желудочным варикозно расширенным венам, обеспечивает полноценную эрадикацию варикозных узлов, способствует уменьшению частоты рецидива кровотечения и летальных исходов.

Ключевые слова: печень, портальная гипертензия, цирроз, пищеводно-желудочное кровотечение, тромбоз, трансъюгулярное внутрипеченочное портосистемное иунтирование, TIPS

Ссылка для цитирования: Хоронько Ю.В., Косовцев Е.В., Козыревский М.А., Хоронько Е.Ю., Криворотов Н.А., Чесноков В.В. Портосистемные шунтирующие операции при осложненной портальной гипертензии: современные возможности мини-инвазивных технологий. Анналы хирургической гепатологии. 2021; 26 (3): 34-45.

https://doi.org/10.16931/1995-5464.2021-3-34-45.

Авторы заявляют об отсутствии конфликта интересов.

\title{
Portosystemic shunting procedures for complicated portal hypertension: modern opportunities of mini-invasive technique
}

Khoronko Yu.V. *, Kosovtsev E.V., Kozyrevskiy M.A., Khoronko E.Yu., Krivorotov N.A., Chesnokov V.V.

Rostov State Medical University, Ministry of Health of the Russian Federation; 29, Nakhichevanskiy str., Rostov-on-Don, 344022, Russian Federation

Aim. To improve the results of treatment for patients with complicated portal hypertension of cirrhotic genesis using the transjugular intahepatic portosystemic shunt with endovascular obliteration of the inflow pathways to the esophageal-gastric varicose veins.

Material and methods. Transjugular intahepatic portosystemic shunt was performed in 172 patients with 
gastroesophageal variceal bleeding. The patients were divided into 3 clinical groups. The shunting procedure was applied to 62 patients. Another 110 patients underwent transjugular intahepatic portosystemic shunt and selective obliteration of the esophageal-gastric vein inflow pathways. The short-term and long-term results (up to 140 months), the incidence of thrombosis, recurrent bleeding and mortality, as well as their relationship with the established risk factors for complications were traced.

Results. All patients achieved an effective reduction in the portosystemic pressure gradient and a reduction in the manifestations of portal hypertension. Recurrence of bleeding caused by shunt thrombosis occurred in 23 (13.3\%) patients. In clinical group I $(n=62)$, this complication was noted in $9(14.5 \%)$ patients, in II $(n=54)-11(20.4 \%)$ cases, in III $(n=56)$ - only in $3(5.4 \%)$ cases. Bleeding-free survival decreased from 1.0 to 0.82 in 83.9 months, then plateaued. A sharp decrease in the Kaplan-Meier curve from 1.0 to 0.88 was observed within 24.5 months after the intervention. The largest number of deaths was in group I (30.6\%), the smallest in group III (7.1\%).

Conclusion. Transjugular intahepatic portosystemic shunt supplemented by obliteration the inflows of the esophagealgastric varicose veins provides complete eradication of varicose veins, helps to reduce the frequency of recurrent bleeding and death.

Keywords: liver, portal hypertension, cirrhosis, gastroesophageal bleeding, thrombosis, transjugular intrahepatic portosystemic shunting

For citation: Khoronko Yu.V., Kosovtsev E.V., Kozyrevskiy M.A., Khoronko E.Yu., Krivorotov N.A., Chesnokov V.V. Portosystemic shunting procedures for complicated portal hypertension: modern opportunities of mini-invasive technique. Annaly khirurgicheskoy gepatologii = Annals of HPB surgery. 2021; 26 (3): 34-45. (In Russian). https://doi.org/10.16931/1995-5464.2021-3-34-45.

There is no conflict of interests.

\section{Введение}

Эффективность портосистемных шунтирующих вмешательств при лечении больных с осложненной портальной гипертензией (ПГ) цирротического генеза у большинства специалистов не вызывает сомнений [1, 2]. Обеспечивая снижение портосистемного градиента давления (ПСГД), эти операции устраняют ПГ - главное звено патогенеза таких угрожающих жизни осложнений, как варикозное пищеводно-желудочное кровотечение (ВПЖК) и рефрактерный асцит (PA), способствующий развитию гепаторенального синдрома [3-5]. Летальность при ВПЖК остается неприемлемо высокой, а меры медикаментозного и эндоскопического гемостаза нередко оказываются малоэффективными [6, 7]. Проведение шунтирующего пособия является ключевым элементом комплекса лечебных мероприятий и позволяет не только сохранить жизнь пациенту, но и увеличить продолжительность так называемого бестрансплантационного периода.

Современное состояние хирургии осложненной ПГ, вызванной циррозом печени (ЦП), характеризуется более широким, чем ранее, внедрением в клиническую практику мини-инвазивных эндоваскулярных вмешательств. К таковым следует отнести в первую очередь трансъюгулярное внутрипеченочное портосистемное шунтирование (Transjugular Intrahepatic Portosystemic Shunt, TIPS) $[1,3,8,9]$, достаточно редко применяемую баллон-окклюзирующую ретроградную трансвенозную облитерацию путей притока к желудочным варикозно расширенным венам (ВРВ; Balloon-occluded Retrograde Transvenous Obliteration, BRTO) и ее модификации [10, 11], a также эндоваскулярную эмболизацию селезеночной артерии, которая утрачивает самостоя- тельное значение [12]. При этом открытые трансабдоминальные шунтирующие вмешательства остаются весьма востребованными [13-16], Следует, однако, подчеркнуть, что их применение считают предпочтительным у больных с сохраненным функциональным резервом печени и компенсированной печеночной недостаточностью $(П Н) ~[7,16]$. Таких пациентов достаточно мало. Специалисты подчеркивают, что состояние большинства больных отягощено суб- или декомпенсированной ПН и выраженными расстройствами системы гемостаза, а варикозная пищеводно-желудочная трансформация зачастую сочетается с асцитом, спленомегалией, гиперспленизмом, печеночной энцефалопатией $[2,4,7,17]$.

Даже безупречное выполнение TIPS и имплантация армированного эндопротеза со специальным покрытием из политетрафторэтилена (РТFE) не всегда позволяют предотвратить тромбоз шунта, который в ранние сроки после вмешательства развивается у 3-25\% оперированных [18, 19]. Результатом тромботической окклюзии шунта является блокирование кровотока по воротной вене (ВВ) и неизбежное возобновление ПГ и переполнение портальной кровью варикозных узлов $[19,20]$. Предотвратить рецидив кровотечения может эндоваскулярная облитерация путей венозного притока к пищеводным и желудочным ВРВ, как предпринятая в качестве самостоятельной хирургической процедуры $[16,21]$, так и дополняющая TIPS [2224]. Детальное изучение ангиоархитектоники левой и задней желудочных вен (ЛЖВ, ЗЖВ) и коротких вен желудка (КВЖ) позволит оптимизировать хирургическую технику селективной облитерации этих сосудов и улучшить результаты TIPS у больных осложненной ПГ. 
Цель исследования - улучшить результаты применения TIPS у больных осложненной ПГ путем эндоваскулярной облитерации сосудов, обеспечивающих приток крови к пищеводножелудочным варикозным узлам.

\section{Материал и методы}

В исследование включены 172 пациента, подвергнутых TIPS по поводу осложненной ПГ цирротического генеза в хирургической клинике ФГБОУ ВО РостГМУ Минздрава России в 20072020 гг. и отвечающих критериям включения. Средний возраст больных - 52,0 $\pm 11,8$ (15-83) года, мужчин было 92 (53,5\%). Критерии включения базировались на показаниях к TIPS. K таковым отнесены недавнее ВПЖК при большом риске рецидива кровотечения $(n=124)$, указание на несколько ВПЖК в анамнезе $(n=27)$, продолжающееся ВПЖК с безуспешным медикаментозным и эндоскопическим гемостазом $(n=8)$, РА в сочетании с выраженным ВРВ пищевода и желудка $(n=13)[3,6]$. Таким образом, в исследование включены больные, которым TIPS предпринято в качестве хирургического пособия для вторичной профилактики варикозного кровотечения или в качестве вмешательства, обеспечивающего эффективную портальную декомпрессию при остром кровотечении (так называемое "раннее" TIPS), а также пациенты с РА и выраженной варикозной трансформацией. Следует заметить, что клиника располагает опытом применения операции TIPS у 221 больного. Показаниями к применению вмешательства пациентам, не отвечавшим критериям включения в исследование, были РА с манифестным или латентным гепаторенальным синдромом 2-го типа $(n=43)$, синдром Бадда-Киари $(n=2)$, посттромботическая трансформация ВВ $(n=4)$.

Причиной ЦП у 95 (55,2\%) больных было поражение вирусами гепатита В или С. Алиментарно-токсическая этиология ЦП установлена у $55(32,0 \%)$ больных. Сочетание вирусного и алкогольного факторов выявлено в 17 (9,9\%) наблюдениях, ахолестаз, приведший к развитию билиарного ЦП, - у $3(1,7 \%)$ пациентов. У 2 больных причина ЦП не установлена. Длительность анамнеза от диагностики ЦП до выполнения шунтирующего хирургического пособия составила $3,3 \pm 2,7$ года (1-140 мес). У всех 172 пациентов заболевание протекало полисиндромно. Среди 159 больных, у которых ведущим, угрожающим жизни проявлением ПГ была варикозная пищеводно-желудочная трансформация, приведшая к кровотечению, у $42(26,4 \%)$ отмечен асцит, у $144(90,6 \%)-$ спленомегалия, у $131(82,4 \%)$ - признаки гиперспленизма. Печеночная энцефалопатия (ПЭ) I стадии, выявленная до TIPS, диагностирована у $24(15,1 \%)$ пациентов, а латентная ПЭ у $83(52,2 \%)$. Соответственно этому у включенных в исследование 13 больных с РА имела место выраженная варикозная пищеводно-желудочная трансформация $(13-100 \%)$. При этом у 12 (92,3\%) из них выявлена спленомегалия с признаками гиперспленизма. ПЭ І стадии была у 3 (23,1\%), а латентная ПЭ - еще у 7 (53,8\%).

У всех 172 пациентов отмечены клинические признаки хронической ПН суб- и декомпенсированной стадий, соответствующих классу В по Child-Pugh у 119 (69,2\%) больных, классу C у $53(30,8 \%)$. Среднее значение $-8,6 \pm 1,6$ балла. Риск планируемой операции оценивали по шкале MELD: <11 баллов, соответствующий низкому риску, установлен у $14(8,1 \%)$ пациентов, 11-17 баллов (умеренный риск) - у 152 $(88,4 \%), 18-24$ (высокий риск) - у 6 (3,5\%). Среднее значение составило $13,1 \pm 3,3$ балла.

172 пациента разделены на три клинические группы. В I (контрольной) группе 62 больным операцию TIPS выполнили без дополнительной облитерации ЛЖВ/ЗЖВ/КВЖ. Во II группе 54 больным TIPS дополнили селективной облитерацией ЛЖВ/ЗЖВ через созданный внутрипеченочный шунт после размещения в нем стента. В III группе 56 пациентам первым этапом выполняли трансъюгулярное формирование внутрипеченочного портосистемного канала, затем селективную облитерацию ЛЖВ/ЗЖВ/КВЖ, после чего завершали TIPS размещением стента в канале. Следует подчеркнуть, что такое распределение соответствовало особенностям ведения пациентов и изменениям в тактике проведения TIPS, происходящим по мере накопления клинического опыта. Для анализа эффективности различных вариантов TIPS у 172 пациентов отслеживали летальные исходы и сроки их наступления, а также тромбоз шунта, сопровождавшийся развитием кровотечения, или без него. Продолжительность наблюдения отдельных пациентов составила 140 мес (11,7 года).

Для исследования выживаемости наибольший период наблюдения (140 мес) был разделен на 30 интервалов, каждый по 4,8 мес. Кумулятивную долю выживших рассчитывали к началу соответствующего временно́го интервала. Анализ выживаемости проводили по методу КапланаМайера. Различия между группами изучены по критерию log-rank теста. Множественное сравнение изменения кумулятивной выживаемости пациентов сразу трех групп осуществлено по критерию Пирсона $\chi^{2}$. Влияние предикторов на риск наступления события изучено с использованием регрессионного анализа Кокса. В качестве предикторов исследовали следующие параметры: число эмболизированных вен и число спиралей, задействованных для получения их облитерации, наличие естественного спленоре- 

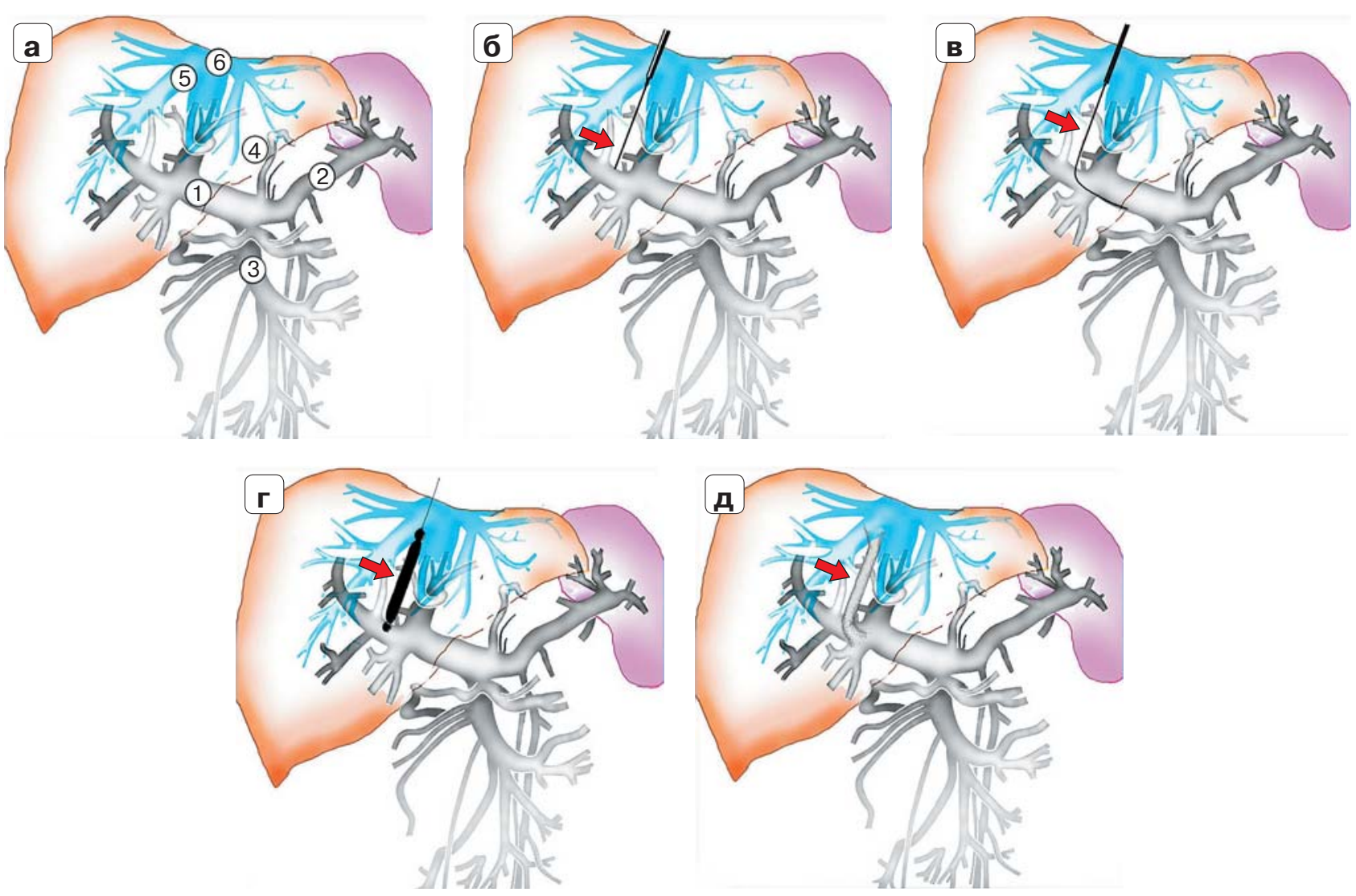

Рис. 1. Этапы TIPS: a - анатомические ориентиры; 1 - ВB, 2 - селезеночная вена, 3 - верхняя брыжеечная вена, 4 - ЛЖВ, 5 - правая печеночная вена, 6 - нижняя полая вена; $\mathbf{\sigma}$ - пункция правой ветви BB иглой Rösch-Uchida (указана красной стрелкой); в - катетеризация ВВ (указана красной стрелкой); г - баллонная дилатация внутрипеченочного портосистемного канала (указан красной стрелкой); д - размещение стента (указан красной стрелкой).

Fig. 1. Stages of transjugular intrahepatic portosystemic shunting: a - anatomical landmarks, 1 - portal vein, 2 - splenic vein, 3 - superior mesenteric vein, 4 - left gastric vein, 5 - right hepatic vein, 6 - inferior vena cave; $\mathbf{0}$ - puncture of the right branch of the with a Rösch-Uchida needle (red arrow); в - catheterization of the portal vein (red arrow); $\mathbf{r}-$ balloon dilatation of the intrahepatic portosystemic canal (red arrow); д - stent placement (red arrow).

нального или портокавального сброса, число стволов ЛЖВ и ЗЖВ, питающих пищеводные и желудочные варикозные узлы, эпизоды смещения стента при вмешательстве. Определяли базальные функции риска для каждого предиктора при его одиночном влиянии на выживаемость пациентов. Далее методами пошагового анализа и многофакторного регрессионного анализа Кокса оценивали одновременное влияние наиболее значимых факторов на риск наступления летального исхода. Оценку сопряжения между летальным исходом пациентов после операции и различными факторами проводили с помощью таблиц сопряженности и критерия $\chi^{2}$ с поправкой Мантеля-Ханзеля.

Техника проведения операции TIPS соответствовала применяемой в большинстве мировых клиник. Как правило, вмешательство осуществляли под местной анестезией с внутривенной безопиоидной анальгезией и седацией на основе пролонгированной дозированной инфузии дексмедетомидина, кетамина и лидокаина. Такая аналгезия и седация хорошо устраняет висцеральную боль, обеспечивает эмоциональный и позиционный комфорт пациенту, а также позволяет сохранить самостоятельное дыхание и при необходимости обратную связь анестезиолога с больным. Основные этапы операции представлены на рис. 1.

При выполнении TIPS до 2015 г. применяли нитиноловые билиарные стенты Shim Hanarostent (покрытый, частично покрытый, чрескожная установка) 8,10 мм и длиной 60,80 мм производства М.І.Тесh (Южная Корея). В 201520 гг. применяли покрытые PTFE стенты Hanarostent Hepatico того же производителя. В качестве эмболизирующих агентов предпочтение отдавали эмболизирующим спиралям модели MReye ${ }^{\circledR}$ (Cook, CША), отличающимся длинными синтетическими волокнами, обеспечивающими значительную тромбогенность (рис. 2). К их достоинствам относят бо́льшую, нежели у платиновых спиралей, радиальную силу. Кроме того, пациенту с имплантирован- 


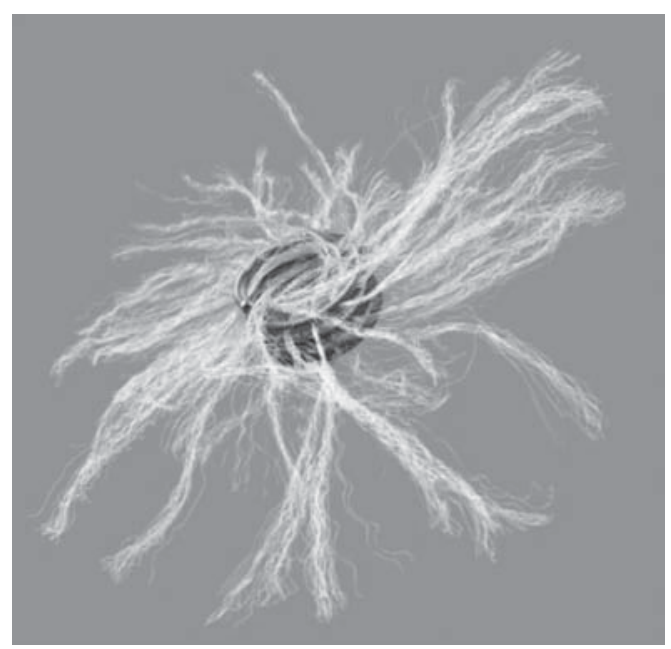

Рис. 2. Эмболизирующая спираль.

Fig. 2. Embolizing coil.

ными спиралями подобного типа не противопоказана МРТ.

Изучены ближайшие и отдаленные результаты TIPS (до 140 мес), выполненного в том числе c селективной облитерацией путей притока к пищеводно-желудочным варикозным узлам, частота тромбоза и рецидивного кровотечения, летальность, а также их связь с установленными факторами риска осложнений.

Клиническому применению селективной облитерации путей притока к пищеводно-желудочным варикозным узлам предшествовало исследование ангиоархитектоники системы ВВ в контексте осуществления эффективной эндоваскулярной эмболизации в дополнение к шунтирующей процедуре TIPS. Материалом послужили серии компьютерных спленопортограмм от 188 больных с ПГ цирротического генеза. Им выполнили МСКТ брюшной полости с сосудистым режимом, обеспечившим получение качественной портовенозной фазы. Для детального изучения путей притока к варикозным узлам, подлежащих эмболизации и последующей облитерации, анализировали серии портограмм, полученных при выполнении TIPS у 202 пациентов на этапе прямой портографии.

\section{Результаты}

Проведение TIPS позволило добиться эффективной портальной декомпрессии у всех 172 пациентов. Это подтверждено снижением показателей давления в ВВ и уровней ПСГД, установленным при прямой манометрии, по сравнению с исходными значениями (таблица).

Клиническим результатом достигнутой портальной декомпрессии стала не только редукция пищеводно-желудочного ВРВ, находящегося в прямой зависимости от уровня ПСГД [3, 9, 16, 18]. Резорбция асцита, выявленного перед TIPS у $42(26,4 \%)$ из 159 пациентов, у которых угрожающим жизни проявлением ПГ была варикозная пищеводно-желудочная трансформация, в течение 6 нед после шунтирующего вмешательства отмечена у 37 (88,1\%). Среди 12 пациентов, у которых ведущим показанием к TIPS был РА, резорбция его в течение 6 нед произошла у 11 (91,7\%). Редукция спленомегалии, выявленной до вмешательства у $156(90,7 \%)$ из 172 оперированных, отмечена в первые 6 нед после TIPS в 145 (92,9\%) наблюдениях. Уменьшилась также выраженность признаков гиперспленизма. Тромбоцитопения, выявленная до операции $\left(61,4 \pm 9,5 \times 10^{9} / л\right)$, по истечении 6 нед с момента формирования TIPS стала менее выраженной $\left(75,8 \pm 10,2 \times 10^{9} / л\right)$. И хотя разница по сравнению с исходными значениями не имела статистической достоверности $\left(p_{\text {исх }}>0,05\right)$, тенденция к редукции гиперспленизма очевидна. Подобная картина прослежена в отношении лейкопении: исходное значение $-3,5 \pm 0,8 \times 10^{9} /$ л, по истечении 6 нед $-4,5 \pm 0,9 \times 10^{9} / л\left(p_{\text {исх }}>0,05\right)$.

По результатам изучения ангиоархитектоники путей притока к пищеводно-желудочным ВРВ установлен ряд фактов, имеющих принципиальное значение в эффективности их полноценной хирургической облитерации и улучшении результатов TIPS. В дополнение к тому, что ЛЖВ принимает участие в формировании пищеводных и желудочных варикозных вен у всех больных, установлено разнообразие как по характеру ее ветвления (от 1 до 4 отходящих от основного ствола ветвей), так и по диаметру

Таблища. Давление в ВB и портосистемный градиент давления до и после TIPS

Table. The pressure in the portal vein and the port-system pressure gradient before and after transjugular intrahepatic portosystemic shunting

\begin{tabular}{l|r|r|r|r|r|r}
\hline \multirow{2}{*}{ Локализация } & \multicolumn{5}{|c}{ Давление, мм рт.ст. } \\
\cline { 2 - 7 } & \multicolumn{2}{|c|}{ І группа } & \multicolumn{2}{c}{ II группа } & \multicolumn{2}{c}{ III группа } \\
\cline { 2 - 7 } & \multicolumn{1}{|c|}{ до TIPS } & \multicolumn{1}{|c}{ после TIPS } & \multicolumn{1}{c|}{ до TIPS } & \multicolumn{1}{c|}{ после TIPS } & \multicolumn{1}{c}{ до TIPS } & после TIPS \\
\hline ВВ & $23,2 \pm 3,1$ & $14,0 \pm 2,7$ & $23,1 \pm 3,2$ & $14,5 \pm 2,8$ & $23,0 \pm 3,5$ & $14,7 \pm 2,7$ \\
ППВ & $5,1 \pm 0,8$ & $7,1 \pm 1,1$ & $5,0 \pm 0,8$ & $7,2 \pm 1,1$ & $5,1 \pm 0,9$ & $7,2 \pm 1,1$ \\
ПСГД & $18,2 \pm 2,9$ & $8,6 \pm 1,1$ & $18,3 \pm 2,7$ & $8,6 \pm 1,0$ & $18,4 \pm 3,3$ & $8,7 \pm 1,1$ \\
\hline
\end{tabular}

Примечание: ВВ - воротная вена; ППВ - правая печеночная вена; ПСГД - портосистемный градиент давления. 

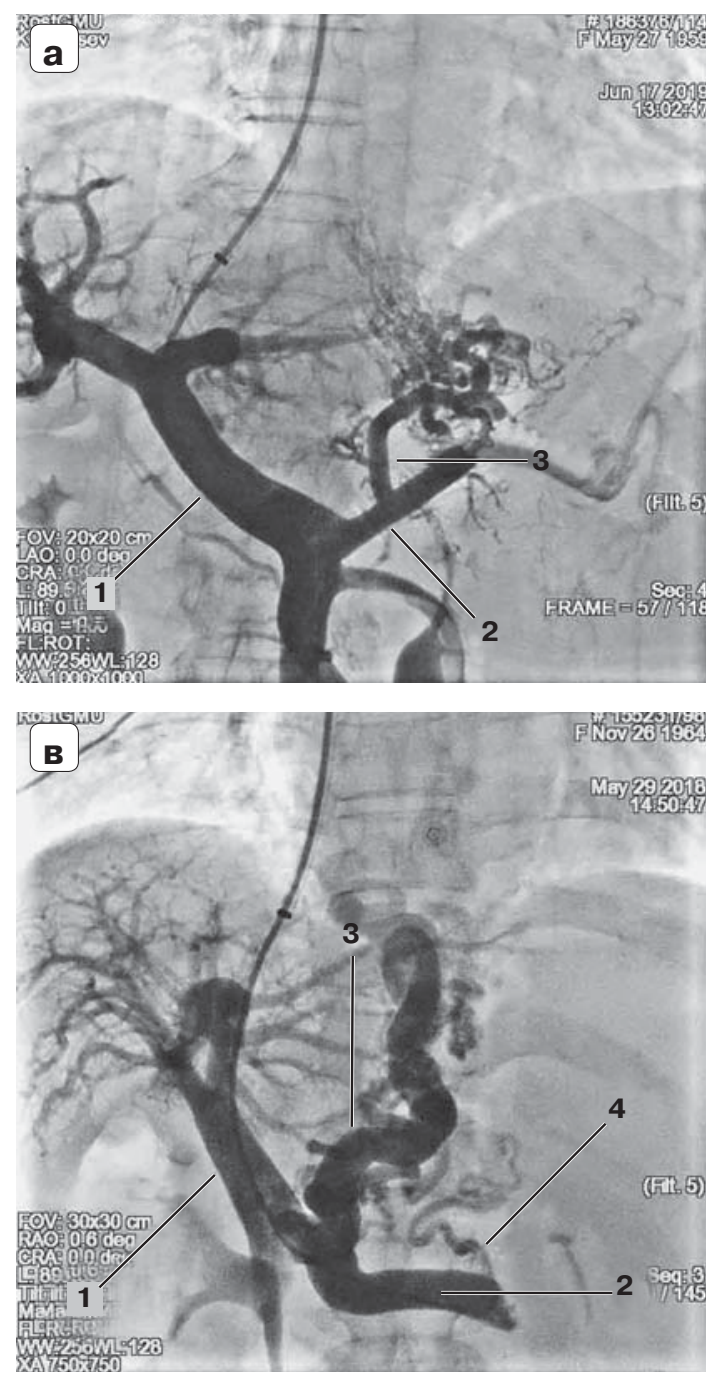
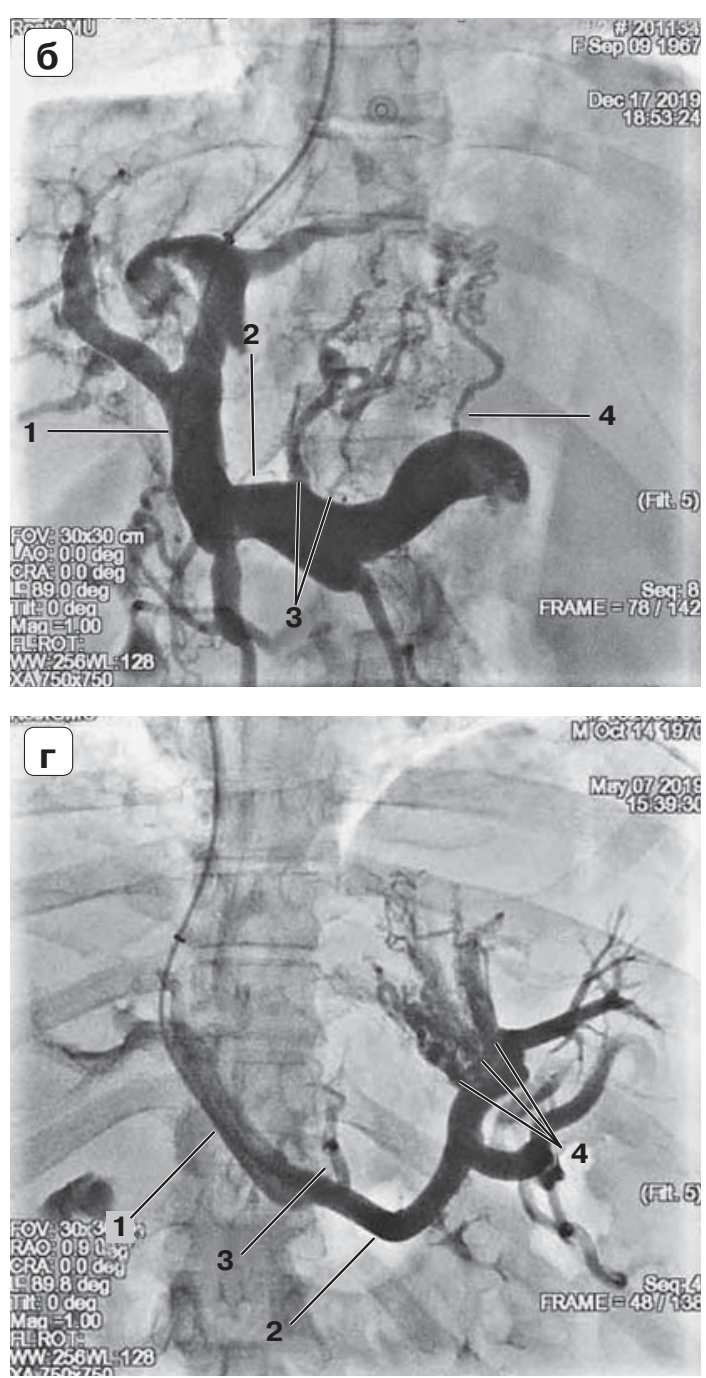

Рис. 3. Интраоперационные портограммы. Варианты ангиоархитектоники ЛЖВ и ЗЖВ, подлежащих эмболизации: $\mathbf{a}-$ солитарная ЛЖВ с выраженным ветвлением; б - удвоенная ЛЖВ и солитарная ЗЖВ; в - солитарный мощный ствол ЛЖВ, превышающий по диаметру селезеночную вену, и тонкая, извитая ЗЖВ; г - ЗЖВ представлена тремя ветвями, а ЛЖВ достаточно тонкая. 1 - ВВ, 2 - селезеночная вена, 3 - ЛЖВ, 4 - ЗЖВ.

Fig. 3. Intraoperative portograms. Variants of the angioarchitectonics of left gastric vein and posterior gastric vein subject to embolization: a - solitary left gastric vein with pronounced branching; $\boldsymbol{\sigma}$ - doubled left gastric vein and solitary posterior gastric vein; в - heavy solitary trunk of left gastric vein, exceeding the splenic vein in diameter and thin, convoluted posterior gastric vein; $\mathbf{r}-$ posterior gastric vein represented by three branches and left gastric vein is quite thin. The numbers indicate: $1-$ portal vein, 2 - splenic vein, 3 - left gastric vein, 4 - posterior gastric vein.

сосуда - от 3 до 12,2 (!) мм (4,8 $\pm 1,4$ мм). Также не установлено прямой корреляционной связи между диаметром ЛЖВ и величиной варикозных узлов. Другой важный сосуд притока - ЗЖВ принимала участие в формировании пищеводных и желудочных ВРВ в 37 (66,1\%) наблюдениях. ЗЖВ, в отличие от ЛЖВ, не является сосудом ангиоархитектоники системы ВВ у здоровых людей, но становится самостоятельным ее элементом лишь при ПГ в качестве весьма значимого коллатерального портосистемного сосудистого пути. Для ЗЖВ, так же как и для ЛЖВ, характерно обширное ветвление. Ветви хаотично переплетаются на пути к варикозным узлам, и в ряде наблюдений их диаметр в области ВРВ значительно больше ствола ЗЖВ. Отмечено, что при селективной ангиографии ЗЖВ вначале контрастируются ВРВ, получающие из нее кровь, затем - пути оттока (нижние диафрагмальные, перикардио-диафрагмальные, непарная и полунепарная вены), а также практически одновременно с ними ветви ЛЖВ (!). Это стало основанием для вывода о существовании анастомозов между ЛЖВ и ЗЖВ, эндоскопически проявляющихся при ПГ выраженной варикозной трансформацией в кардиофундальной зоне. Помимо ЛЖВ и ЗЖВ, изредка в качестве пути притока к желудочным ВРВ выявляют КВЖ. Прикладное значение полученных результатов заключается в обязательной эндоваскулярной эмболизации всех установленных путей притока к пищеводно-желудочным ВРВ, а не только ЛЖВ (рис. 3). 

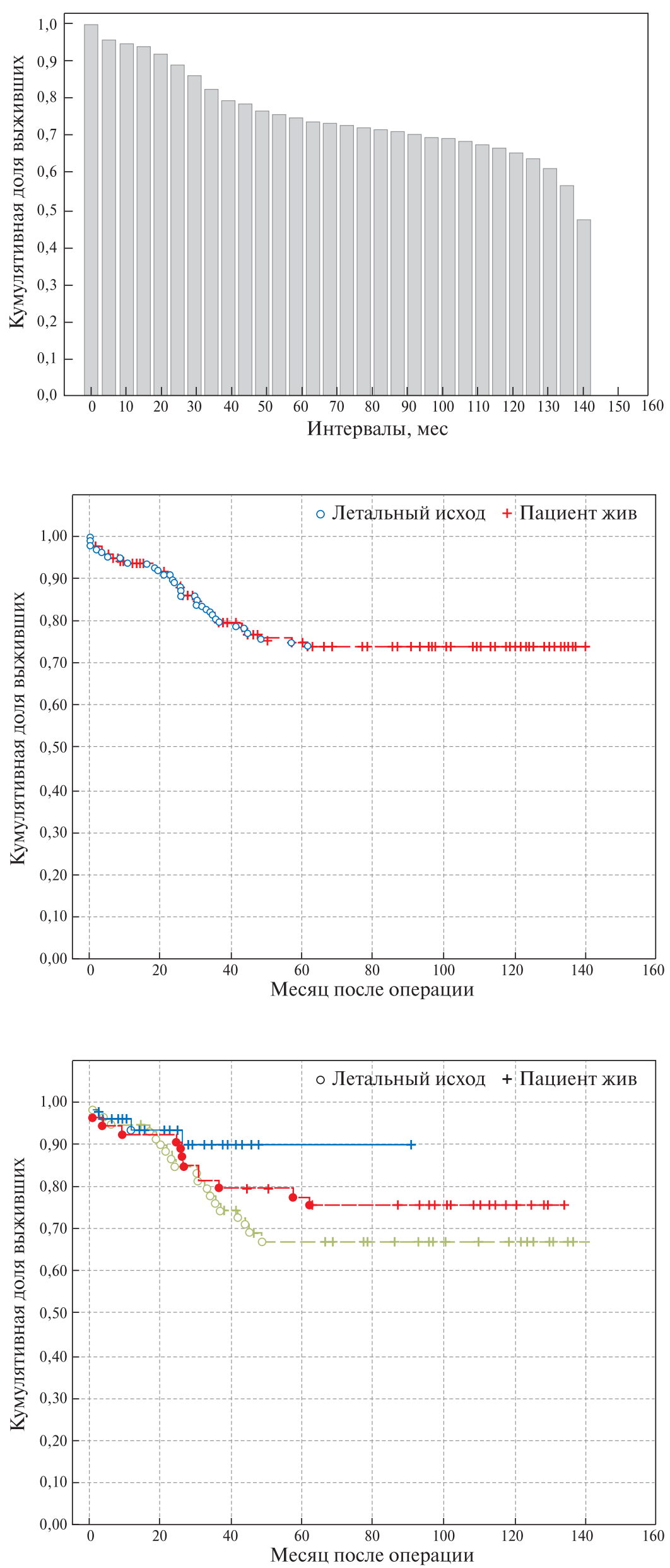

Рис. 4. Диаграмма. Кумулятивная выживаемость пациентов.

Fig. 4. Diagram. Cumulative patient survival.

Рис. 5. Диаграмма. Общая выживаемость по Каплану-Майеру.

Fig. 5. Diagram. Overall survival rate according to Kaplan-Meier.
Рис. 6. Диаграмма. Выживаемость по Каплану-Майеру в группах исследования.

Fig. 6. Diagram. Survival according to Kaplan-Meier in study groups. 
Процедура облитерации путей притока к варикозным узлам в дополнение к шунтирующему этапу TIPS не приводила к увеличению продолжительности операции. Средняя ее продолжительность в I группе составила 73,31 мин, во II - 79,46 мин, в III - 70,11 мин $(p>0,05)$. Продолжительность рентгеноскопии (мин) и значения эквивалентной дозы ионизирующего излучения (мЗв) составили соответственно в I группе 21,57 мин и 21,14 м3в, во II - 23,1 мин и 22,82 м3в, в III - 21,74 мин и 22,67 м3в $(p>0,05)$.

На протяжении всего периода наблюдений рецидивные кровотечения, вызванные тромбозом шунта, произошли у $23(13,3 \%)$ пациентов. При этом в I клинической группе осложнение отмечено у 9 (14,5\%) больных, во II группе у $11(20,4 \%)$, а в III - лишь у $3(5,4 \%)$. Число тромбозов шунта и рецидивных кровотечений статистически значимо различалось только между II и III группами $(p=0,038)$. Бо́льшая частота рецидива в I группе по сравнению с III объясняется тем, что при дисфункции портосистемного шунта возобновляется патологический кровоток к пищеводно-желудочным варикозным узлам. Большое число рецидивов кровотечения во II группе даже по сравнению с I группой вызвано тем, что у больных II группы не удавалось осуществить полноценную эмболизацию всех путей притока к ВРВ: эффективно блокировать удавалось лишь ЛЖВ, а кровоток через ЗЖВ сохранялся и даже компенсаторно возрастал.

Отслежены летальные исходы и сроки их наступления, изучена выживаемость пациентов. Максимальная продолжительность наблюдения составила 140 мес (11,7 лет; рис. 4). Угрожающие жизни сосудистые события (тромбоз шунта, кровотечение) отмечены у $23(13,4 \%)$ пациентов. Выживаемость без кровотечения уменьшалась с 1,0 до 0,82 за 83,9 мес, а затем переходила в плато. Резкое снижение кривой КапланаМайера наблюдали с 1,0 до 0,88 за 24,5 мес, или в первые 2 года после операции (рис. 5). Наибольшее число летальных исходов отмечено в I группе (30,6\%), наименьшее - в III группе $(7,1 \%)$. Во II группе отмечено $13(24,1 \%)$ летальных исходов. Множественное сравнение числа летальных исходов в трех группах выявило статистически значимое различие $(p=0,006)$. В III группе показатель достоверно меньше по сравнению со II $(p=0,028)$ и I группами $(p=0,003)$. Через 31 мес $(2,6$ года) после операции между группами впервые сформировалось различие кумулятивной доли выживших (рис. 6).

В III группе $(89,8 \%)$ показатель выживаемости был наибольшим, более выраженное уменьшение числа выживших пациентов произошло во II группе (до 83,3\%) и I группе $(81,5 \%)$. B III группе начиная с этого интервала кумуля- тивная доля выживших не уменьшалась и оставалась на уровне $89,8 \%$, во II группе она уменьшилась до 75,8\% и в I группе - до 67\%. По критерию $\log$-rank теста различие между II и III группами $(p=0,043)$, I и III группами $(p=0,022)$ было статистически значимым. Это позволило утверждать, что облитерация ЛЖВ и ЗЖВ наиболее эффективно увеличивает выживаемость пациентов в той группе, в которой эта процедура предшествовала стентированию внутрипеченочного портосистемного канала.

\section{Обсуждение}

Внедрение мини-инвазивных технологий для портосистемных шунтирующих операций у больных с осложненной ПГ цирротического генеза отражает современные тенденции развития гепатобилиарной хирургии. TIPS занимает весьма заметное место в комплексе лечебных мероприятий у больных с суб- и декомпенсированной ПН, которым показано портосистемное шунтирование в связи с ВПЖК или большим риском его рецидива, а также при РА и гепаторенальном синдроме 2-го типа. Эффективность TIPS подтверждена редукцией пищеводно-желудочной варикозной трансформации у пациентов всех трех групп настоящего клинического исследования, а также положительной динамикой резорбции асцита и уменьшением выраженности таких проявлений ПГ, как спленомегалия и гиперспленизм. Следует признать, что очевидные достоинства TIPS соседствуют с недостатками, среди которых видное место занимает вероятность тромбоза рукотворного портосистемного шунта, нередко возникающего на фоне выраженных расстройств системы гемостаза у пациентов с ЦП. Развитие дисфункции шунта приводит к нарастанию ПСГД, восстановлению патологического коллатерального портосистемного кровотока, переполнению пищеводно-желудочных ВРВ и возобновлению роли рисков, связанных с ПГ.

Изучение ангиоархитектоники путей притока портальной крови к пищеводным и желудочным варикозным узлам дало основание полагать, что их хирургическое блокирование может способствовать уменьшению риска рецидива кровотечения при тромбозе сформированного при TIPS внутрипеченочного шунта. Достичь этого можно дополнением шунтирующего этапа вмешательства селективной эмболизацией ЛЖВ и ЗЖВ, а при необходимости - и КВЖ для их облитерации.

Техническая осуществимость облитерации путей притока к ВРВ венам продемонстрирована как во II, так и в III группе. Установлено, что при выполнении TIPS пациентам II группы, когда первым этапом во внутрипеченочном портосистемном канале размещали стент, а затем эмболи- 
зировали пути притока к ВРВ, у хирурга отсутствовала возможность полноценного осуществления манипуляций по селективной катетеризации ЛЖВ и ЗЖВ. Затруднения были вызваны риском смещения уже установленного стента. По результатам оценки динамики выживаемости установлено, что увеличение эффективности TIPS у больных с осложненной ПГ цирротического генеза обеспечивается селективной эмболизацией ЛЖВ, ЗЖВ и при необходимости - КВЖ первым этапом, предшествующим стентированию внутрипеченочного шунта, что применено у пациентов III группы исследования.

Наконец, необходимо подчеркнуть, что эффективность TIPS в полной мере может быть обеспечена лишь соблюдением определенного алгоритма ведения больного, подвергнутого данному вмешательству. Хорошо известно, что прогнозируемым осложнением вмешательства является развитие энцефалопатии. Для ее профилактики ключевое внимание уделяли следующим назначениям: обеспечению ежедневного стула, препараты лактулозы 30-60 мл/сут per os, рифаксимин 800-1200 мг/сут per os, курсами продолжительностью 5-7 сут ежемесячно, диетические ограничения - отказ от алкоголя, уменьшение потребления животного белка.

\section{Заключение}

TIPS является эффективным мини-инвазивным портосистемным шунтирующим пособием, удовлетворительно переносимым пациентами с осложненным течением ПГ на фоне суб- и декомпенсированной ПН цирротического генеза. У больных с ПГ, осложненной большим риском ВПЖК, TIPS целесообразно сочетать с облитерацией путей притока к пищеводным и желудочным ВРВ, представленным ЛЖВ, ЗЖВ и КВЖ. Хирургическое блокирование этих притоков, дополняющее эффективную портальную декомпрессию TIPS, способствует более полноценной ликвидации варикозных узлов, уменьшению риска рецидивного кровотечения и улучшению выживаемости пациентов.

\section{Участие авторов}

Хоронько Ю.В. - концепция исследования, научное руководство, написание текста.

Косовцев Е.В. - дизайн исследования, статистическая обработка данных.

Козыревский М.А. - ответственность за целостность всех частей статьи; анатомические исследования.

Хоронько Е.Ю. - редактирование; статистическая обработка данных.

Криворотов Н.А. - сбор и обработка материала.

Чесноков В.В. - сбор и обработка материала.

Все авторы принимали участие в обсуждении результатов и формировании заключительной версии статьи.

\section{Authors participation}

Khoronko Yu.V. - concept of the study, scientific leadership, writing text.

Kosovtsev E.V. - design of the study, statistical analysis.

Kozyrevskiy M.A. - responsibility for the integrity of all parts of the article, anatomical studies.

Khoronko E.Yu. - editing, statistical analysis.

Krivorotov N.A. - collection and analysis of data.

Chesnokov V.V. - collection and analysis of data.

All authors discussed the results and contributed in the final manuscript.

\section{Список литературы}

1. Затевахин И.И., Шиповский В.Н., Цициашвили М.Ш., Монахов Д.В. Портальная гипертензия: диагностика и лечение. Практическое руководство. М.: Буки Веди, 2015. $328 \mathrm{c}$.

2. Simonetto D.A., Liu M., Kamath P.S. Portal hypertension and related complications: diagnosis and management. Mayo Clin. Proc. 2019; 94 (4): 714-726.

https://doi.org/10.1016/j.mayocp.2018.12.020

3. Шерцингер А.Г., Чжао А.В., Ивашкин В.Т., Маевская М.В., Павлов Ч.С., Верткин А.Л., Огурцов П.П., Лопаткина Т.Н., Котив Б.Н., Дзидзава И.И., Анисимов А.Ю., Прудков М.И., Хоронько Ю.В., Назыров Ф.Г., Девятов А.В., Киценко Е.А. Лечение кровотечений из варикозно расширенных вен пищевода и желудка. Анналы хирургической гепатологии. 2013; 18 (3): 110-129.

4. Манукьян Г.В., Шерцингер А.Г. Дифференцированное хирургическое лечение больных циррозом печени с портальной гипертензией. Часть I. Оценка тяжести течения заболевания и выбор метода хирургического вмешательства. Анналы хирургической гепатологии. 2015; 2 (1): 10-13. https://doi.org/10.16951/1995-5464.2018476-85

5. Liu C., Liu Y., Wang S., Wang G., Wang R., Zhang M., Hou J., Zhang Ch., Qi X. The predictive value of base line hepatic venous pressure gradient for variceal rebleeding in cirrhotic patients receiving secondary prevention. Ann. Transl. Med. 2020; 8 (4): 91. https://doi.org/10.21037/atm.2019.12.143

6. Жигалова С.Б., Манукьян Г.В., Шерцингер А.Г., Фандеев Е.Е., Семенова Т.С., Коршунов И.Б., Мартиросян Р.А. Прогностические критерии кровотечений из варикозно расширенных вен пищевода и желудка у больных портальной гипертензией. Анналы хирургической гепатологии. 2018; 23 (4): 76-85. https://doi.org/10.16951/1995-5464.2018476-85

7. Garcia-Tsao G., Abraldes J.G., Berzigotti A., Bosch J. Portal hypertensive bleeding in cirrhosis: risk stratification, diagnosis, and management: 2016 practice guidance by the American Association for the Study of Liver Diseases. Hepatology. 2017; 65 (10): 310-335. https://doi.org/10.1002/hep.28906

8. García-Pagán J.C., Saffo S., Mandorfer M., Garcia-Tsao G. Where does TIPS in the management of patients with cirrhosis? JHEP Rep. 2020; 2 (4): 100-122.

https://doi.org/10.1016/j.jhepr.2020.100122

9. Rajesh S., George T., Philips C.A., Ahamed R., Kumbar S., Mohan N., Mohanan M., Augustine P. Transjugular intrahepatic portosystemic shunt in cirrhosis: an update exhaustive critical update. World J. Gastroenterol. 2020; 26 (37): 5561-5596. https://doi.org/10.3748/wjg.v26.i37.5561 
10. Zhu Y., Wang X., Luo X., Yang L. Balloon-occluded retrograde transvenous obliteration (BRTO) or coil-assisted retrograde transvenous obliteration (CARTO): which one do we choose? Am. J. Gastroenterol. 2018; 113 (12): 1901-1902.

https://doi.org/10.1038/s41395-018-0228-Z

11. Lipnik A.J., Pandhi M.B., Khabbaz R.C., Gaba R.C. Endovascular treatment for variceal hemorrhage: TIPS, BRTO, and combined approaches. Semin. Intervent. Radiol. 2018; 35 (3): 169-184. https://doi.org/10.1055/s-0038-1660795

12. Ishikawa T., Sasaki R., Nishimura T., Aibe Y., Saeki I., Iwamoto T., Hidaka I., Takami T., Sakaida I. A novel therapeutic strategy for esophageal varicesusing endoscopic treatment combined with splenicartery embolization according to the Child-Pugh classification. PLoS One. 2019; 14 (9): e0223153. https://doi.org/10.1371/journal.pone.0223153

13. Котив Б.Н., Дзидзава И.И., Солдатов С.А., Кашкин Д.П., Алентьев С.А., Смородский А.В., Слободяник А.В., Онинцев И.Е. Результаты селективного и парциального портокавального шунтирования и прогностические факторы долгосрочной выживаемости больных циррозом печени. Анналы хирургической гепатологии. 2015; 20 (2): 46-58. https://doi.org/10.16931/1995-5464.2015246-58

14. Назыров Ф.Г., Девятов А.В., Бабаджанов А.Х. Результаты и перспективы портосистемного шунтирования у больных циррозом печени. Анналы хирургической гепатологии. 2015; 20 (2): 31-39. https://doi.org/10.16931/1995-5464.2015231-39

15. Brand M., Prodehl L., Ede C.J. Surgical portosystemic shunts versus transjugular intrahepatic portosystemic shunt for variceal haemorrhage in people. Cochrane Database Syst. Rev. 2018; 2018 (10): CD001023. https://doi.org/10.1002/14651858. CD001023.pub3

16. Glowka T.R., Kalff J.C., Manekeller S. Update on shunt surgery. Visc.Med.2020;36(3):206-211.https://doi.org/10.1159/000507125

17. Ивашкин В.Т., Маевская М.В., Павлов Ч.С., Федосьина Е.А., Бессонова Е.Н., Пирогова И.Ю., Гарбузенко Д.В. Клинические рекомендации Российского общества по изучению печени и Российской гастроэнтерологической ассоциации по лечению осложнений цирроза печени. Российский журнал гастроэнтерологии, гепатологии, колопроктологии. 2016; 26 (4): 71-102. https://doi. org/10.22416/1382-4376-2016-26-4-71-102

18. Затевахин И.И., Цициашвили М.Ш., Шиповский В.Н., Монахов Д.В., Челяпин А.С. Опыт повторных вмешательств при нарушении проходимости портокавальных шунтов, выполненных методом TIPS. Хирургия. Журнал им. Н.И. Пирогова. 2019; 6: 11-19. https://doi.org/10.1711/ hirurgia201906111

19. Lv Y., Han G., Fan D. Thrombosis after transjugular intrahepatic portosystemic shunt: an ominous sign? AME Med. J. 2017; 2: 40-43. https://doi.org/10.21037/amj.2017.03.07

20. Jahangiri Y., Kerrigan T., Li L., Prosser D., Brar A., Righetti J., Schenning R.C., Kaufman J.A., Farsad K. Risk factors for stent graft thrombosis after transjugular intrahepatic portosystemic shunt creation. Cardiovasc. Diagn. Ther. 2017: 7 (3): 150-158. https://doi.org/10.21037/cdt.2017.10.03

21. Khera P.S., Myungsu L., Joonsung C. Balloon occluded retrograde transvenous obliteration for bleeding gastric varices: eyes see what the mind knows. Indian J. Radiol. Imaging. 2017; 27 (1): 100-104. https://doi.org/10.4103/0971-3026.202952

22. Gaba R.C. Transjugular intrahepatic portosystemic shunt creation with embolization or obliteration for variceal bleeding.
Tech. Vasc. Interv. Radiol. 2016: 19 (1): 21-35.

https://doi.org/10.1053/j.tvir.2016.01.003

23. Schultheiß M., Giesler M., Maruschke L., Schmidt A., Sturm L., Thimme R., Rössle M., Bettinger D. Adjuvant transjugular variceal occlusion at creation of a transjugular intrahepatic portosystemic shunt (TIPS): efficacy and risks of bucrylate embolization. Cardiovasc. Intervent. Radiol. 2019; 42 (5): 729736. https://doi.org/10.1007/s00270-019-02176-y

24. Yu J., Wang X., Jiang M., Ma H., Zhou Z., Yang L., Li X. Comparison of transjugularintra hepatic portosystemic shunt (TIPS) alone and combined with embolisation for the management of cardiofundal varices: a retrospective study. Eur. Radiol. 2019; 29 (2): 699-706.

https://doi.org/10.1007/s00330-018-5645-2

\section{References}

1. Zatevakhin I.I., Shipovskii V.N., Tsitsiashvili M.Sh., Monakhov D.V. Portal'naya gipertenziya: diagnostika i lechenie. Prakticheskoe rukovodstvo [Portal hypertension: diagnosticsand treatment]. Moscow: BukiVedi, 2015. 328 p. (In Russian)

2. Simonetto D.A., Liu M., Kamath P.S. Portal hypertension and related complications: diagnosis and management. Mayo Clin. Proc. 2019; 94 (4): 714-726.

https://doi.org/10.1016/j.mayocp.2018.12.020

3. Shertsinger A.G., Chzhao A.V., Ivashkin V.T., Maevskaya M.V., Pavlov Ch.S., Vertkin A.L., Ogurtsov P.P., Lopatkina T.N., Kotiv B.N., Dzidzava I.I., Anisimov A.Yu., Prudkov M.I., Khoronko Yu.V., Nazyrov F.G., Devyatov A.V., Kitsenko E.A. Treatment of variceal esophageal and gastric bleeding. Annaly khirurgicheskoy gepatologii = Annals of HPB Surgery. 2013; 18 (3): 110-129. (In Russian)

4. Manukyan G.V., Shertsinger A.G. Differentiated surgical treatment of portal hypertension and its complications in patients with liver cirrhosis. Part I. Assessment of diseases severity and choice of surgical intervention. Annaly khirurgicheskoy gepatologii = Annals of HPB Surgery. 2015; 2 (1): 10-13. https:// doi.org/1016931/1995-5464.2018476-85 (In Russian)

5. Liu C., Liu Y., Wang S., Wang G., Wang R., Zhang M., Hou J., Zhang Ch., Qi X. The predictive value of base line hepatic venous pressure gradient for variceal rebleeding in cirrhotic patients receiving secondary prevention. Ann. Transl. Med. 2020; 8 (4): 91. https://doi.org/10.21037/atm.2019.12.143

6. Zhigalova S.B., Manukiyan G.V., Shertsinger A.G., Fandeev E.E., Semenova T.S., Korshunov I.B., Martirosyan R.A. Prognostic criteria of variceal bleeding in patients with portal hypertension. Annaly khirurgicheskoy gepatologii $=$ Annals of HPB Surgery. 2018; 23 (4): 76-85. https://doi.org/10.16951/1995-5464.2018476-85 (In Russian)

7. Garcia-Tsao G., Abraldes J.G., Berzigotti A., Bosch J. Portal hypertensive bleeding in cirrhosis: risk stratification, diagnosis, and management: 2016 practice guidance by the American Association for the Study of Liver Diseases. Hepatology. 2017; 65 (10): 310-335. https://doi.org/10.1002/hep.28906

8. García-Pagán J.C., Saffo S., Mandorfer M., Garcia-Tsao G. Where does TIPS in the management of patients with cirrhosis? JHEP Rep. 2020; 2 (4): 100-122.

https://doi.org/10.1016/j.jhepr.2020.100122

9. Rajesh S., George T., Philips C.A., Ahamed R., Kumbar S., Mohan N., Mohanan M., Augustine P. Transjugular intrahepatic portosystemic shunt in cirrhosis: an update exhaustive critical update. World J. Gastroenterol. 2020; 26 (37): 5561-5596. https://doi.org/10.3748/wjg.v26.i37.5561 
10. Zhu Y., Wang X., Luo X., Yang L. Balloon-occluded retrograde transvenous obliteration (BRTO) or coil-assisted retrograde transvenous obliteration (CARTO): which one do we choose? Am. J. Gastroenterol. 2018; 113 (12): 1901-1902.

https://doi.org/10.1038/s41395-018-0228-Z

11. Lipnik A.J., Pandhi M.B., Khabbaz R.C., Gaba R.C. Endovascular treatment for variceal hemorrhage: TIPS, BRTO, and combined approaches. Semin. Intervent. Radiol. 2018; 35 (3): 169-184. https://doi.org/10.1055/s-0038-1660795

12. Ishikawa T., Sasaki R., Nishimura T., Aibe Y., Saeki I., Iwamoto T., Hidaka I., Takami T., Sakaida I. A novel therapeutic strategy for esophageal varicesusing endoscopic treatment combined with splenicartery embolization according to the Child-Pugh classification. PLoS One. 2019; 14 (9): e0223153. https://doi.org/10.1371/journal.pone.0223153

13. Kotiv B.N., Dzidzava I.I., Soldatov S.A., Kashkin D.P., Alentiev S.A., Smorodskiy A.V., Slobodyanik A.V., Onintsev I.E. Results of the selective and partial portocaval bypass and prognostic factors of long-term survival in patients with liver cirrhosis. Annaly khirurgicheskoy gepatologii = Annals of HPB Surgery. 2015; 20 (2): 46-58. https://doi.org/10.16931/19955464.2015246-58 (In Russian)

14. Nazyrov F.G., Devyatov A.V., Babadzhanov A.K. Results and prospects of portosystemic shunting in patients with liver cirrhosis. Annaly khirurgicheskoy gepatologii $=$ Annals of $H P B$ Surgery. 2015; 20 (2): 31-39. https://doi.org/10.16931/19955464.2015231-39 (In Russian)

15. Brand M., Prodehl L., Ede C.J. Surgical portosystemic shunts versus transjugular intrahepatic portosystemic shunt for variceal haemorrhage in people. Cochrane Database Syst. Rev. 2018; 2018 (10): CD001023. https://doi.org/10.1002/14651858. CD001023.pub3

16. Glowka T.R., Kalff J.C., Manekeller S. Update on shunt surgery. Visc.Med.2020;36(3):206-211.https://doi.org/10.1159/000507125

17. Ivashkin V.T., Maevskaya M.V., Pavlov Ch.S., Fedosyina E.A., Bessonova E.N., Pirogova I.Yu., Garbuzenko D.V. Treatment of liver cirrhosis complications: Clinical guidelines of the Russian Scientific Liver Society and Russian Gastroenterological
Association. Russian Journal of Gastroenterology, Hepatology, Coloproctology. 2016; 26 (4): 71-102. https://doi. org/10.22416/1382-4376-2016-26-4-71-102 (In Russian)

18. Zatevakhin I.I., Tsitsiashvili M.Sh., Shipovskii V.N., Monakhov D.V., Chelyapin A.S. Redo surgery for portocaval shunt dysfunction after TIPS procedure (in Russian only). Pirogov Russian Journal of Surgery $=$ Khirurgiya. Zhurnal imeni N.I. Pirogova. 2019; 6: 11-19. https://doi.org/10.1711/ hirurgia201906111 (In Russian)

19. Lv Y., Han G., Fan D. Thrombosis after transjugular intrahepatic portosystemic shunt: an ominous sign? AME Med. J. 2017; 2: 40-43. https://doi.org/10.21037/amj.2017.03.07

20. Jahangiri Y., Kerrigan T., Li L., Prosser D., Brar A., Righetti J., Schenning R.C., Kaufman J.A., Farsad K. Risk factors for stent graft thrombosis after transjugular intrahepatic portosystemic shunt creation. Cardiovasc. Diagn. Ther. 2017: 7 (3): 150-158. https://doi.org/10.21037/cdt.2017.10.03

21. Khera P.S., Myungsu L., Joonsung C. Balloon occluded retrograde transvenous obliteration for bleeding gastric varices: eyes see what the mind knows. Indian J. Radiol. Imaging. 2017; 27 (1): 100-104. https://doi.org/10.4103/0971-3026.202952

22. Gaba R.C. Transjugular intrahepatic portosystemic shunt creation with embolization or obliteration for variceal bleeding. Tech. Vasc. Interv. Radiol. 2016: 19 (1): 21-35. https://doi.org/10.1053/j.tvir.2016.01.003

23. Schultheiß M., Giesler M., Maruschke L., Schmidt A., Sturm L., Thimme R., Rössle M., Bettinger D. Adjuvant transjugular variceal occlusion at creation of a transjugular intrahepatic portosystemic shunt (TIPS): efficacy and risks of bucrylate embolization. Cardiovasc. Intervent. Radiol. 2019; 42 (5): 729736. https://doi.org/10.1007/s00270-019-02176-y

24. Yu J., Wang X., Jiang M., Ma H., Zhou Z., Yang L., Li X. Comparison of transjugularintra hepatic portosystemic shunt (TIPS) alone and combined with embolisation for the management of cardiofundal varices: a retrospective study. Eur. Radiol. 2019; 29 (2): 699-706.

https://doi.org/10.1007/s00330-018-5645-2

\section{Сведения об авторах [Authors info]}

Хоронько Юрий Владиленович - доктор мед. наук, профессор, заведующий кафедрой оперативной хирургии и топографической анатомии, врач-хирург хирургического отделения ФГБОУ ВО РостГМУ Минздрава России.

http://orcid.org/0000-0002-3752-3193. E-mail: khoronko507@gmail.com

Косовцев Евгений Валерьевич - канд. мед. наук, заведующий отделением рентгенохирургических методов диагностики и лечения ФГБОУ ВО РостГМУ Минздрава России. http://orcid.org/0000-0001-8547-1001. E-mail: kosovtsev@yandex.ru

Козыревский Михаил Александрович - канд. мед. наук, ассистент кафедры оперативной хирургии и топографической анатомии, врач-хирург хирургического отделения ФГБОУ ВО РостГМУ Минздрава России. ORCID: 0000-00029652-1710. E-mail: kozyrevskiy@mail.ru

Хоронько Евгений Юрьевич - канд. мед. наук, ассистент кафедры хирургических болезней №1, врач-хирург хирургического отделения ФГБОУ ВО РостГМУ Минздрава России. http://orcid.org/0000-0001-5261-9620. E-mail: khoronko081@mail.ru Криворотов Николай Анатольевич - врач-хирург хирургического отделения ФГБОУ ВО РостГМУ Минздрава России. http://orcid.org/0000-0002-8759-5654. E-mail: krivokolk@mail.ru

Чесноков Владимир Владимирович - клинический ординатор кафедры хирургических болезней №2 ФГБОУ ВО РостГМУ Минздрава России. http://orcid.org/0000-0001-6800-9894. E-mail: vladimirchesnokov@gmail.com

Для корреспонденции *: Хоронько Юрий Владиленович - 344022, Ростов-на-Дону, пер. Нахичеванский, д. 29. Тел.: +7-938-100-04-83. E-mail: khoronko507@gmail.com 
Yuriy V. Khoronko - Doct. of Sci. (Med.), Professor, Head of the Department of Operative Surgery and Clinical Anatomy, Rostov State Medical University, Ministry of Health of the Russian Federation. http://orcid.org/0000-0002-3752-3193. E-mail: khoronko507@gmail.com

Evgeniy V. Kosovtsev - Cand. of Sci. (Med.), Head of the Radiology Department, Rostov State Medical University, Ministry of Health of the Russian Federation. http://orcid.org/0000-0001-8547-1001. E-mail: kosovtsev@yandex.ru

Michail A. Kozyrevskiy - Cand. of Sci. (Med.), Assistant of the Department of Operative Surgery and Clinical Anatomy, Rostov State Medical University, Ministry of Health of the Russian Federation. http://orcid.org/0000-0002-9652-1710. E-mail: kozyrevskiy@mail.ru

Evgeniy Yu. Khoronko - Cand. of Sci. (Med.), Assistant of the Department of Surgical Diseases No.1, Rostov State Medical University, Ministry of Health of the Russian Federation. http://orcid.org/0000-0001-5261-9620. E-mail: khoronko081@mail.ru Nikolay A. Krivorotov - Surgeon of the Department of Surgery, Rostov State Medical University, Ministry of Health of the Russian Federation. http://orcid.org/0000-0002-8759-5654.E-mail: krivokolk@mail.ru

Vladimir V. Chesnokov - Resident of the Department of Surgical Diseases No.2, Rostov State Medical University, Ministry of Health of the Russian Federation. http://orcid.org/0000-0001-6800-9894. E-mail: vladimirchesnokov@gmail.com

For correspondence*: Yuriy V. Khoronko - Department of Operative Surgery and Clinical Anatomy, Rostov State Medical University, Rostov-on-Don. Phone: +7-938-100-04-83. E-mail: khoronko507@gmail.com

Статья поступила в редакцию журнала 11.05.2021. Received 11 May 2021.
Принята к публикации 1.06.2021. Accepted for publication 1 June 2021. 